\title{
Spontaneous Emission Enhancement of CdSe Quantum Dots Embedded in a Two-dimensional Photonic Crystal L3 Nanocavity
}

\author{
Khalid N. Sediq \\ Department of Physics, Koya University, Faculty of Science and Health, Koya University, University Park, \\ Danielle Mitterrand Boulevard, Koya KOY45, Kurdistan Region - F.R. Iraq
}

\begin{abstract}
Two-dimensional photonic crystal nanocavities were designed to tailor cavity quantum electrodynamics. Enhancing the spontaneous emission of low-quality factor nanocavity with embedded CdSe quantum dots (QDs) emitters is the aim of this study. Low concentration layer of CdSe QDs was sandwiched between two layers of $\mathrm{Si}_{2} \mathrm{~N}_{3}$ membrane using plasma-enhanced chemical vapor deposition. The modification rate in spontaneous emission of $L 3$ nanocavity up to 2.3 -fold has been observed at $629.5 \mathrm{~nm}$ in compare to bare cavities. High field confinement in the sub-wavelength regime became an interest field for quantum electrodynamics applications and good platform to study light matter interactions.
\end{abstract}

Index Terms-Quantum dots; L3 cavity; Photonic crystal; Purcell factor.

\section{INTRODUCTION}

The ability to confine light into small volumes is important for enhancing the light and matter interaction in the emerging field of nanophotonics. Optical confinement in the sub-wavelength regime is of high interest for quantum electrodynamics applications (Chang, et al., 2006; Gong and Vučković, 2007). They are bounding the electromagnetic excitations between semiconductors and a dielectric, leading to a strong interaction between quantum emitters and photon fields (Chang, et al., 2006). This interaction can further be enhanced through an appropriate feedback mechanism that provided by nanocavity structures. The enhancement of cavity spontaneous emission (Purcell effect) is essential for a variety of applications, such as, single-photon sources (Wie, 2019), integrated quantum optics (Mahmoodian, et al., 2016; Daveau, et al., 2017), nanoscale lasers (Oulton, et al., 2009), active meta-materials (Schulz, et al., 2016), biotechnological devices for enhanced fluorescence intensity (Lakowicz, et

ARO-The Scientific Journal of Koya University

Vol. IX, No.2 (2021), Article ID: ARO.10810, 4 pages DOI: $10.14500 /$ aro. 10810

Received: 21 April 2021; Accepted: 07 October 2021

Regular research paper: Published: 02 November 2021

Corresponding author's email: khalid.sediq@koyauniversity.org Copyright (C) 2021 Khalid N. Sediq. This is an open-access article distributed under the Creative Commons Attribution License. al., 2003), and ultra-fast modulated LEDs (Shambat, et al., 2011). Nanolaser performances are also highly affected by light emitters that introduced within the resonator. The gain properties of semiconductor quantum dots (QDs) have a lot of advantageous due to their temperature stability, small line width enhancement factor, and the ability to suppress the nonradiative recombination (Arakawa, et al., 1982). In addition, QDs are suitable for studying cavity QED, because of their atom like quantum emission (Yoshie, et al., 2004; Reithmaier, et al., 2004; Arakawa, et al., 2012). Recently, a study has been carried on field improvement at communication wavelength using InGaAs QDs embedded in a GaAs nanowire having groove like cavity, which manifested high enhancement by 617-fold (Wie, 2019). Recently, some studies were carried out on field improvement at communication wavelength, for example, InGaAs QDs was embedded in a GaAs nanowire having groove like cavity, which manifested high enhancement by 617-fold (Wie, 2019); while using Ge/Si QDs within $\mathrm{Si}$ two-dimensional photonic crystal (2D PC) field enhanced by about 25 times at $\lambda=1.2 \mu \mathrm{m}$ and by 34 times $\lambda=1.6 \mu \mathrm{m}$ (Yakimov, 2021).

In this study, field enhancement toward visible wavelength using CdSe QDs embedded in $\mathrm{Si}_{2} \mathrm{~N}_{3} 2 \mathrm{D}$ PC was proposed. A remarkable field enhancement was observed using even low-quality factor L3 nanocavity. Such result can be promising to study light mater interaction at visible wavelength.

\section{MATERIALS AND METHODS}

In this work, the realization and characterization of a hybrid photonic crystal (PC) cavity that combines the benefits of both QDs and photonic elements has been presented. Here, an $8 \mathrm{~nm}$ of CdSe QDs (provided by Sigma-Aldrich) is used as an active emitter layer.

\section{A. 2D PC Fabrication}

First, a suspended layer of $\mathrm{Si}_{2} \mathrm{~N}_{3}$ with a thickness of $100 \mathrm{~nm}$ was coated with a thin layer of $8 \mathrm{~nm}$ of CdSe QDs which diluted to a concentration of $0.01 \mathrm{mg} / \mathrm{ml}$ in toluene solution. Second, another layer with $100 \mathrm{~nm}$ of $\mathrm{Si}_{2} \mathrm{~N}_{3}$ is deposited by plasma-enhanced chemical vapor deposition. The whole 
structure thickness becomes $200 \mathrm{~nm}$ with a sandwiched layer of CdSe QDs. A $200 \mathrm{~nm}$ polymethyl-methacrylate (PMMA) electron beam resist layer was spin coated on the $\mathrm{Si}_{2} \mathrm{~N}_{3}$ surface then backed on a hot plate for $1 \mathrm{~min}$ at $100^{\circ} \mathrm{C}$ to harden it and remove any residual solution. Subsequently, a $30 \mathrm{kV}$ electron beam lithography was used to define the required PC pattern in PMMA. Then, exposed PC pattern in resist by electron beam is removed by methyl isobutyl ketone: isopropyl alcohol 1:3 developer. Finally, the PC pattern is transferred from PMMA into the $\mathrm{Si}_{2} \mathrm{~N}_{3}$ layer using reactive ion etching to obtain air holes through the $\mathrm{Si}_{2} \mathrm{~N}_{3}$ layer. The used recipe of the RIE dry etching device was based on a mixture gas of $\mathrm{CHF}_{3}$ and $\mathrm{O}_{2}$, with flow rates 30 and $5 \mathrm{sccm}$, respectively.

\section{B. Photolumens (PL) Measurements}

The fabricated PC is optically pumped using a CW diode pumped solid state laser at $532 \mathrm{~nm}$. The beam focused through a $100 \times$ long working distance objective lens $(6 \mathrm{~mm})$ having a 0.77 numerical aperture (NA) to a $2.5 \mu \mathrm{m}$ diameter spot on the cavity surface. The laser spot was controlling using a three-dimensional piezo motor stage (Piezosystem Jena Tritor 100, Micro XYZ Positioner stage NV 40/3s controller). The emission spectra were collected using the same objective lens and analyzed by nitrogen cooled CCD detector. Long-pass filter with a cutoff wavelength at $532 \mathrm{~nm}$ was used to block the excitation light from reaching the detector.

\section{Results AND Discussion}

The QDs solution diluted to a concentration of $0.01 \mathrm{mg} / \mathrm{ml}$ in toluene and spun into the structures at $1.5 \mathrm{krpm}$ (to reduce unwanted aggregation) on $100 \mathrm{~nm} \mathrm{Si}{ }_{2} \mathrm{~N}_{3}$ layer, resulting up to $\sim 10^{2}$ emitters dots per $2 \mu \mathrm{m}^{2}$. The coating was not uniform due to the small chip size of the membrane $\left(0.25 \mathrm{~mm}^{2}\right)$ as it is shown in Fig. 1.

PL has been taken for embedded layer of QDs as shown in Fig. 2. The emission spectrum shows a peak position at $640 \mathrm{~nm}$ with full width at half maximum (FWHM) $50 \mathrm{~nm}$. The wide width of emission spectrum line is due to the effect of the dots aggregation which leads to red shift, besides the influence $\mathrm{Si}_{2} \mathrm{~N}_{3}$ layer PL emission.

The fabricated PC dimension is $10 \mu \mathrm{m} \times 10 \mu \mathrm{m}$ with three missed holes in the center act as nanocavity. Such structure is known as PC with L3 cavity. The produced PC L3 cavity has a lattice constant $\mathrm{a}=260 \mathrm{~nm}$ (distance between two adjacent holes), hole radius $r=0.288 \mathrm{a}$, and total cavity length $0.78 \mu \mathrm{m}$ approximately, as shown in Fig. 3.

In general, $\mathrm{PC}$ as an optical system can enhance or suppress the rates of spontaneous emission for any quantum emitters. The coupling between cavity modes and the QDs as twolevel system is a type of energy exchanging between them. In such system (QDs - nanocavity), photons are supplied with a certain probability that depends on their environment and density of states. The coupling between quantum emitters and cavity mode leads to a remarkable enhancement of the spontaneous emission of the output modes which is known as Purcell effect (Purcell, 1946) and the Purcell factor is totally

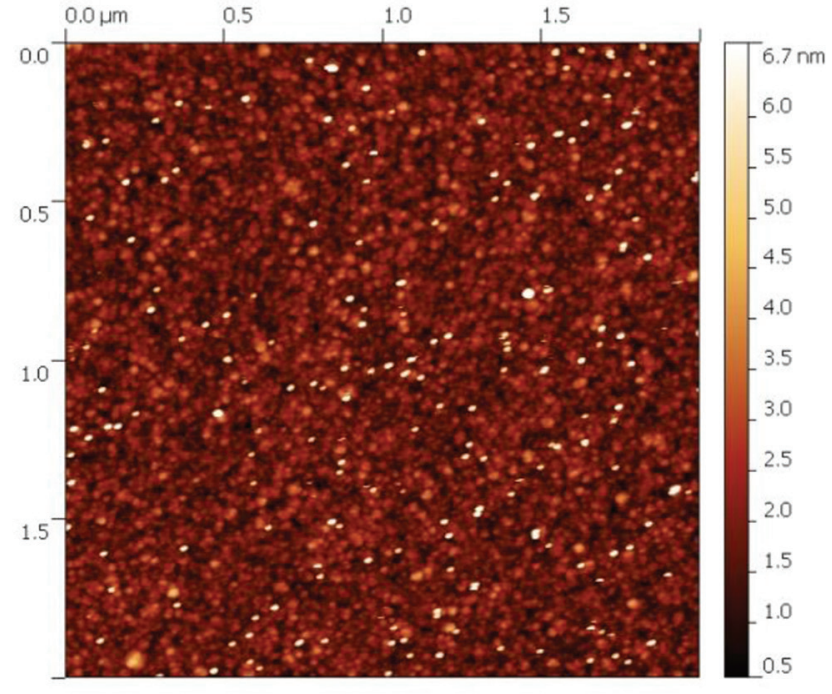

Fig. 1. Atomic force microscopy image of thin layer of $0.01 \mathrm{mg} / \mathrm{mL}$ of CdSe quantum dots coated on a thin layer of $\mathrm{Si}_{2} \mathrm{~N}_{3}$ membrane. Acceptable distribution and low aggregation are observed.

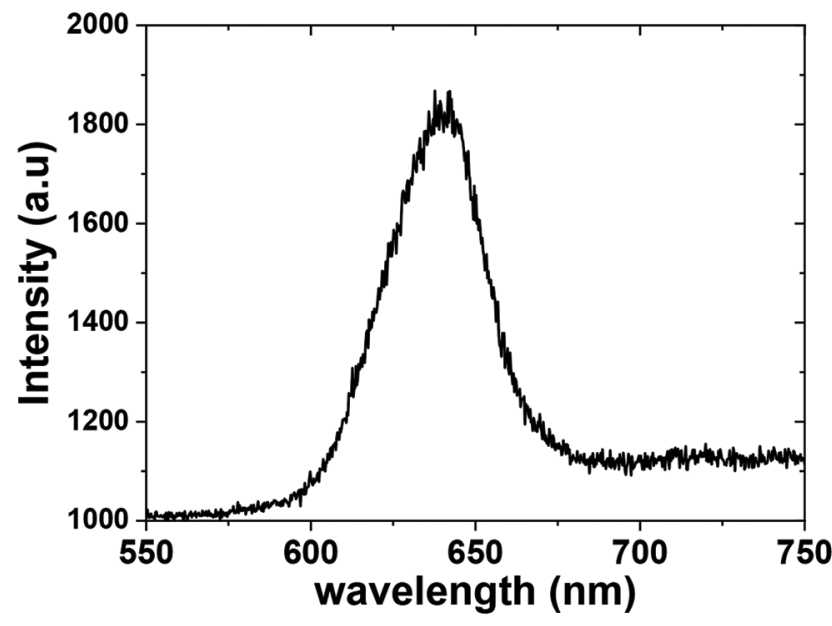

Fig. 2. Photo lumens emission of $0.01 \mathrm{mg} / \mathrm{mL}$ of CdSe quantum dots sandwiched within $200 \mathrm{~nm}$ layer of $\mathrm{Si}_{2} \mathrm{~N}_{3}$ layer.

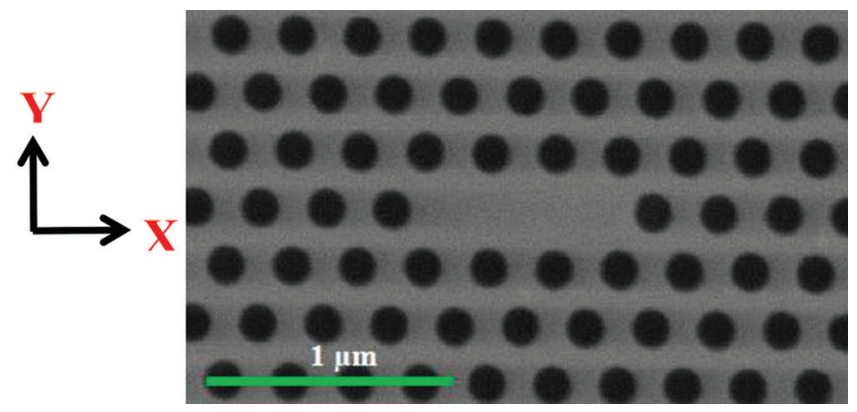

Fig. 3. Scanning electron microscope image of two-dimensional photonic crystal $\mathrm{L} 3$ cavity printed on $\mathrm{Si}_{2} \mathrm{~N}_{3}$ membrane.

depend on $\mathrm{Q} / \mathrm{V}$ ratio (Ryu and Notomi, 2003):

$$
F=\frac{3}{4 \pi^{2}} \frac{Q}{V_{\text {mode }}}\left(\frac{\lambda_{0}}{n}\right)^{3}
$$


Where: $\mathrm{Q}$ quality factor, $\mathrm{V}_{\text {mode }}$ mode volume, and $\mathrm{n}$ is medium refractive index.

The enhancement of spontaneous emission rate $\gamma$ is strongly ruled by the emitter alignment with the dipole moment $\vec{\mu}_{i}$ at a point on the cavity, which can be derived from Fermi's Golden rule (Boroditsky, et al., 1999; Englund, et al., 2005).

$$
\gamma=\frac{\gamma_{\text {cav }}}{\gamma_{\text {free }}}=F \times\left(\frac{\overrightarrow{E \cdot \vec{\mu}}}{|\vec{E}|_{\text {max }}|\vec{\mu}|}\right)^{2} \times \frac{\Delta \lambda_{c a v}^{2}}{4\left(\lambda-\lambda_{\text {cav }}\right)^{2}+\Delta \lambda_{c a v}^{2}}
$$

To measure the enhancement of the confined field through Purcell factor inside nanocavity before and after introducing QDs emitters, number of parameters should be estimated, such as quality factor $\mathrm{Q}$ and mode volume $\mathrm{V}_{\text {mode }}$.

Physically, the effective cavity mode volume is known as a spatial distribution of electromagnetic energy (Balanis, 2013).

$$
V_{\text {mode }}=\frac{\int \in E^{2} d V}{\max \left(\in E^{2}\right)}
$$

In purely dielectric cavities, the ratio of the volume of the active region $\left(\mathrm{V}_{\mathrm{a}}\right)$ to mode volume $\left(\mathrm{V}_{\text {mode }}\right)$ is known as confinement factor (Chang and Chuang, 2009):

$$
\tilde{\mathrm{A}}=\frac{V_{a}}{V_{\text {mode }}}
$$

Therefore, to obtain high confined cavities, the mode volume should be smaller than the active region volume, that is, $\Gamma>1$. In general, the 3D nanocavity effective mode volume calculation needs to use numerical methods due to the geometric and material complexity of the cavities. Experimentally, the modes are considered completely confined to the active region volume; $\mathrm{V}_{\text {mode }}$ approaches the geometrical active medium volume $\mathrm{V}_{\mathrm{a}}$ and Equation 4 becomes $\Gamma \approx 1$ (Li, 2018).

In this experiment, cavities without QDs show no output mode enhancement (Fig. 4). However, PC L3 designed cavity supports one mode on $\mathrm{X}$-axis direction (long cavity axis), $\lambda=625 \mathrm{~nm}$ FWHM $\Delta \lambda=2.08 \mathrm{~nm}$ (Fig. 5 red curve). Mode quality factor $\mathrm{Q}$ inside nanocavity which known as the ratio of stored energy to dissipated one can be calculated by $Q=\lambda \Delta \lambda$. Hence, mode cavity shows low-quality factor $\mathrm{Q}=303$ (Fig. 5 red curve) whereas the field enhancement calculated from Purcell factor from Eq. 1 is $F_{\text {bare }}=30.29$ using the mode volume normalized to the mode wavelength $V_{\text {mode }}=0.76\left(\frac{\lambda}{n}\right)^{3}$.

Introducing a thin layer of CdSe QDs which sandwiched in the PC membrane, two modes were observed $\lambda_{1}=624.5 \mathrm{~nm}$ and $\lambda_{2}=629.5 \mathrm{~nm}$ with both $\Delta \lambda \approx 0.9 \mathrm{~nm}$ as shown in Fig. 5 (black curve). Consequently, both modes' Q-factor increased significantly to $Q_{1}=690$ and $Q_{2}=699$, respectively. Using the same mode volume normalized to the mode wavelength in the Purcell factor formula for the highest $\mathrm{Q}_{2}$, factor mode

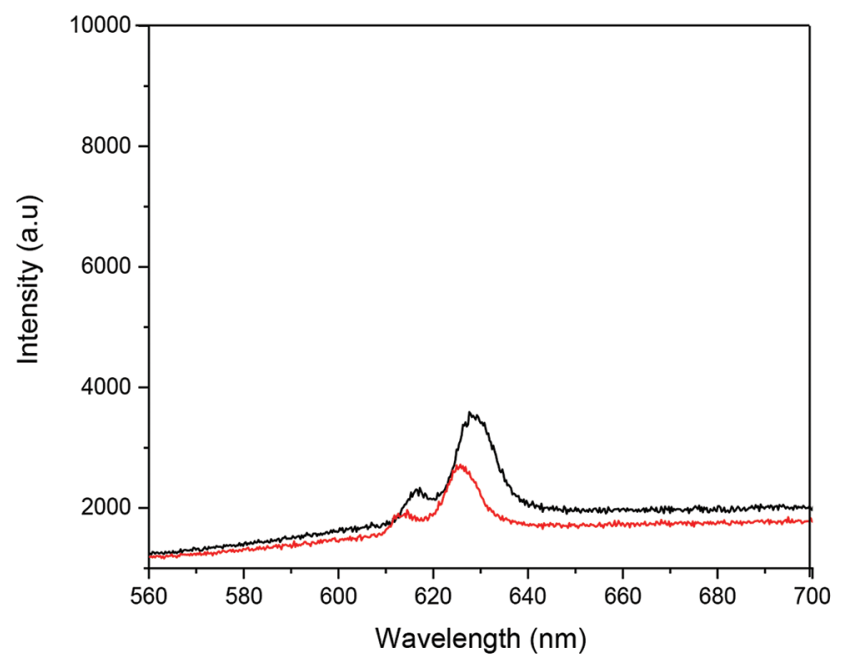

Fig. 4. Photo lumens spectrum for L3 cavity modes before introducing CdSe quantum dots (QDs) (red curve) and after introducing a thin layer of CdSe QDs (black curve).

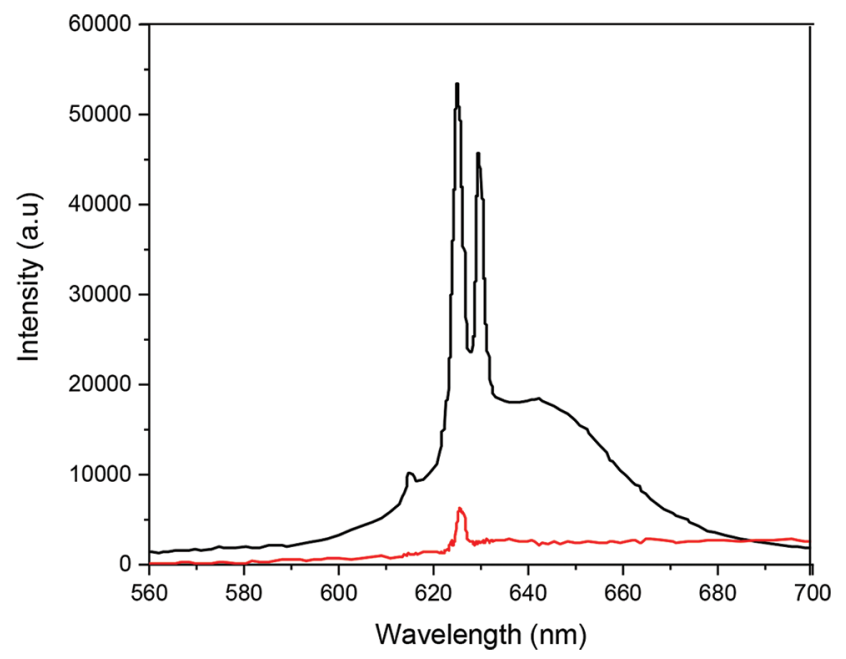

Fig. 5. Photo lumens (PL) spectrum for L3 cavity modes before introducing CdSe quantum dots (QDs) (red curve) and after introducing a thin layer of CdSe QDs (black curve). Here, QDs emission modes are highly aligned with cavity modes shows remarkable enhancement of PL.

is $\mathrm{F}_{\mathrm{QD}}=71.29$ so that the cavity field shows enhancement by 2.36-fold $\left(\mathrm{F}=\mathrm{F}_{\mathrm{QD}} / \mathrm{F}_{\text {bare }}\right)$.

In addition, Eq. 2 indicates that the location of quantum emitters inside nanocavities plays an essential role to either enhance or suppress the output emission. Thus, the PC cavity modes-QD exciton system on resonance should be located at the maxima electric cavity field (Vuckovic and Yamamoto, 2003). Enhancements in both $\lambda 1$ and $\lambda 2$ modes (Fig. 5) can be referred to the increase of coupling parameter to the decay rates ratio through strong coupling system (Vuckovic and Yamamoto, 2003; Yoshie, et al., 2001).

On the other hand, cavities that contain slightly misaligned QDs position show PL enhancement but low quality factor mode $\mathrm{Q}=233$ as illustrated in Fig. 6 (red curve before and black curve after introducing QDs layer). 


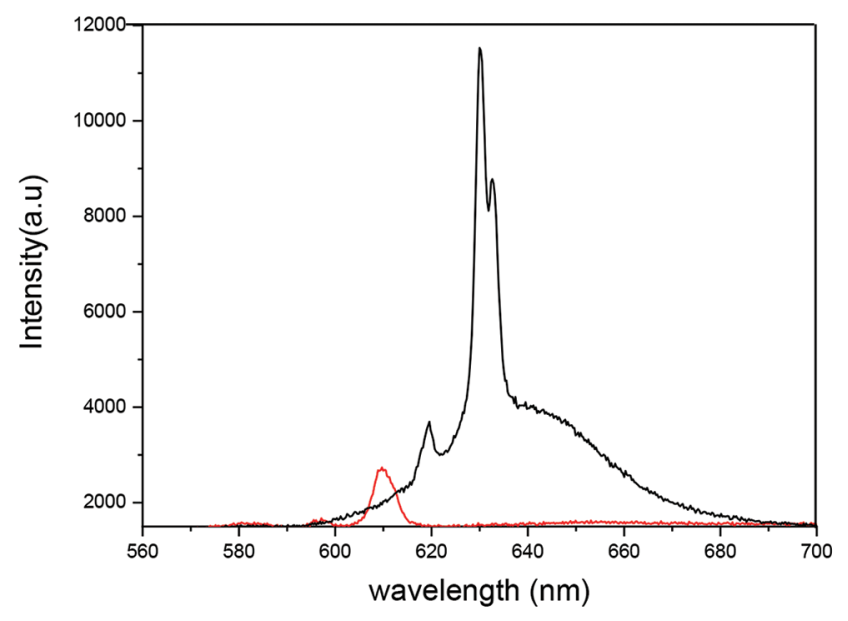

Fig. 6. Photo lumens (PL) spectrum for L3 cavity modes before introducing CdSe quantum dots (QDs) (red curve) and after introducing a thin layer of CdSe QDs (black curve). Here, QDs emission modes are slightly misaligned with cavity modes. PL emission shows enhancement but low Q factor 233.

\section{SUMMERY}

PC cavity-QD emitters system is a good platform to study light matter interaction. Mode field enhancement was observed in the order of 2.3-fold at $629.5 \mathrm{~nm}$ which occurs when the QD and the cavity are in resonant and the QD is spatially aligned to the cavity mode. The advantages of the used technique are the low cost and limitation of using chemicals, however, it is difficult to locate QDs at a desirable position due to random distribution of the QDs during the coating process. Using semiconductor, QDs as a gain material can improve the effective light matter interactions through nanolasers performance.

\section{REFERENCES}

Arakawa, Y. and Sakaki, H., 1982. Multidimensional quantum well laser and temperature dependence of its threshold current. Applied Physics Letters, 40(11), pp.939-941.

Arakawa, Y., Iwamoto, S., Nomura, M., Tandaechanurat, A. and Ota, Y., 2012. Cavity quantum electrodynamics and lasing oscillation in single quantum dotphotonic crystal nanocavity coupled systems. IEEE Journal of Selected Topics in Quantum Electronics, 18(6), pp.1818-1829.

Balanis, C.A., 2012. Advanced Engineering Electromagnetics, $2^{\text {nd }}$ ed. John Wiley and Sons Comp., Hoboken, NJ, USA.

Boroditsky, M., Vrijen, R., Krauss, T., Coccioli, R., Bhat, R. and Yablonovitch, E., 1999. Spontaneous emission extraction and purcell enhancement from thin-film 2-D photonic crystals. Journal of Light Wave Technolegy, 17(11), p.6424901.

Chang, E., Sorensen, S., Hemmer, R. and Lukin, D., 2006. Quantum optics with surface plasmons. Physical Review Letters, 97, p.053002.

Chang, S. and Chuang, S.L., 2009. Fundamental formulation for plasmonic nanolasers. IEEE Journal of Quantum Electronics, 45(11), pp.1014-1023.
Daveau, R., Balram, K., Pregnolato, T., Liu, J., Lee, E., Song, J., Verma, V., Mirin, R., Nam, S., Midolo, L., Stobbe, S., Srinivasan, K. and Lodahl, P., 2017. Efficient fiber-coupled single-photon source based on quantum dots in a photoniccrystal waveguide. Optica, 4(2), pp.178-184.

Englund, D., Fattal, D., Waks, E., Solomon, G., Zhang, B., Nakaoka, T., Arakawa, Y., Yammamoto, Y. and Vučković, J., 2005. Controlling the spontaneous emission rate of single quantam dots in two dimentional photonic crystal. Physical Review Letters, 95(1), pp.013904-013908.

Gong, Y. and Vučković, J., 2007. Design of plasmon cavities for solid-state cavity quantum electrodynamics applications. Applied Physics Letters, 90, p. 033113.

Lakowicz, J., Malicka, J., Gryczynski, I., Gryczynski, Z. and Geddes, C., 2003. Radiative decay engineering: The role of photonic mode density in biotechnology. Journal of Physics D, 36(14), p.R240-R249.

Li, X., Smalley, J., Li, Z. and Gu, Q., 2018. Effective modal volume in nanoscale photonic and plasmonic near-infrared resonant cavities. Applied Sciences, 8, p.1464.

Mahmoodian, S., Lodahl, P., and Sørensen, A., 2016. Quantum networks with chiral-light-matter interaction in waveguides. Physical Review Letters, 117, p. 240501 .

Oulton, R., Sorger, V., Zentgraf, T., Ma, R., Gladden, C., Dai, L., Bartal, G. and Zhang, X., 2009. Plasmon lasers at deep sub-wavelength scale. Nature, 461 , pp. 629-632.

Purcell, E., 1948. Spontaneous emission probabilities at radio frequencies. Physical Review, 69, p.681.

Reithmaier, J., Sek, J., Loffler, A., Hofmann, C., Kuhn, S., Reitzenstein, S., Keldysh, L., Kulakovskii, V., Reinecke, T. and Forchel, A., 2004. Strong coupling in a single quantum dot-semiconductor microcavity system. Nature, 432(7014), pp.197-200.

Ryu, Y. and Notomi, M., 2003. Enhancement of spontaneous emission from the resonant modes of a photonic crystal slab single-defect cavity. Optics Express, 28(23), pp.2390-2392.

Schulz, K., Vu, H., Schwaiger, S., Rottler, A., Korn, T., Sonnenberg, T., Kipp, T. and Mendach, S., 2016. Controlling the spontaneous emission rate of quantum wells in rolled-up hyperbolic metamaterials. Physical Review Letters, 117, p.085503.

Shambat, G., Ellis, B., Majumdar, A., Petykiewicz, J., Mayer, M., Sarmiento, T., Harris, J., Haller, E. and Vučković, J., 2011. Ultrafast direct modulation of a single-mode photonic crystal nanocavity light-emitting diode. Nature Communions, 2, p.539.

Vuckovic, J. and Yamamoto, Y., 2003. Photonic crystal microcavities for cavity quantum electrodynamics with a single quantum dot. Applied Physics Letters, 82(15), p.2374.

Wei, W., Yan, X., Liu, J., Shen, B., Luo, W., Ma, X. and Zhang, X., 2019. Enhancement of single-photon emission rate from InGaAs/GaAs quantum-dot/ nanowire heterostructure by Wire-Groove nanocavity. Nanomaterials (Basel, Switzerland), 9(5), p.671.

Yakimov, I., Kirienko, V., Bloshkin A., Utkin, E. and Dvurechenskii, V., 2021. Near-infrared photoresponse in Ge/Si quantum dots enhanced by photon-trapping hole arrays. Nanomaterials, 11(9), p.2302.

Yoshie, T., Scherer, A., Hendrickson, J., Khitrova, G., Gibbs, H., Rupper, G., Ell, C., Shchekin, O. and Deppe, D., 2004. Vacuum Rabi splitting with a single quantum dot in a photonic crystal nanocavity. Nature, 432, pp.200-203.

Yoshie, T., Vuc ${ }^{` k o v i c, ~ J . ~ a n d ~ S c h e r e r, ~ A ., ~ 2001 . ~ H i g h ~ q u a l i t y ~ t w o-d i m e n s i o n a l ~}$ photonic crystal slab cavities. Applied Physics Letters, 79(26), p.4289. 